\title{
Radio frequency Echo mapping with cellular devices
}

\author{
Isahar Gabay ${ }^{*}$ (D, Meir Danino and Zeev Zalevsky
}

\begin{abstract}
In this paper, a method is presented that employs portable cellular devices to perform three-dimensional localization of metallic objects from a set of time-delayed signals. These signals are observed by an array of receiving elements having arbitrary and a priori known distributions in space. Widely used solutions for direction finding are based on Time of Arrival (TOA) measurements. The measurement accuracy is strongly dependent on several system and scenario parameters, such as the target relative distance, number of received elements, distances between elements, azimuth and elevation beam-widths, all of which may cause ambiguity and multi-path effects. It is important that operational systems are capable of working with both static and moving targets. A recent solution involves obtaining Time Difference of Arrival (TDOA) measurements. To improve the accuracy of the measurements, a Delta Delta Phase (DDPhase) technique is herein proposed. It is suitable for even slow-moving targets, such as the one used in this study. Experimental results showed that the presented solution is feasible and with an accuracy of up to $0.3 \mathrm{~m}$ for detecting targets, including those occluded behind a concrete wall.
\end{abstract}

Keywords: Antenna arrays, Delta-delta phase, Monopulse, Radio frequency, Time difference of arrival, Time of arrival, Threedimensional mapping

\section{Introduction}

A key task of direction finding systems is determination to the arrival direction of targets. As the most stable parameter of the targets, the arrival direction plays an important role as a sorting parameter. The task of instantaneous direction finding (Demmel, 2009; Gething, 1990; Lim, Chae, \& Park, 2005) is very challenging because the signal may possess one of many frequencies within an extremely wide frequency band in all directions and in dense emitter environments. Consequently, many types of instantaneous direction finding systems, such as the scanning beam (Saunders, 1999), amplitude comparison (Mailloux, 2005), phased interferometer or array (Brookner, 1991; Hansen, 2010; Mailloux, 2005), TOA (Grosicki \& Abed Meraim, 2005; Tuncer \& Friedlander, 2009), and Time Difference of Arrival (TDOA) (Patwari et al., 2003) have been developed.

Monopulse radars are used to track the positions of moving targets. They avoid problems related to the power fluctuations of the back-scattered signal (i.e., echo) due to the finite amount of time in which the target is illuminated

\footnotetext{
* Correspondence: gabayi@elta.co.il

Faculty of Engineering, Bar-llan University, Max Vana Wob St., Ramat-Gan, Israel
}

by the transmitted pulse by using short-term observation of the target echo and comparing the so-called sum and difference signals (Rocca \& Morabito, 2015).

The methods cited above enable metallic reflectors (such as a rifles) to be located even if they are within concrete walls. They achieve this by comparing the reflected RF signal to the source signal, both of which are detected by different sensors that are carefully distributed near the scene. As walls and doors are transparent to the RF radiation, they do not affect the detection. The data from the different sensors are collected with accurate profile and timing (0.1-0.5 ns) for each pulse, and are analyzed in real time to ascertain the respective locations of the source and metallic reflector.

In this study, the methods cited above were analyzed and found to be unsuitable for the current purpose. Therefore, a method is herein proposed the DDPhase approach that is applicable not only to the subject scenario, but also to moving targets (even at high speeds). This method is suitable for direct LOS obstruction cases, such as targets occluded behind concrete walls. This is the first time that this method is presented in professional papers or books. This method is a result of practical designing and 
commercial research of direction finding systems in tactical operational. There is not a motivation to compared this method to all of other previous methods as mentioned above, but it is compared to the last famous one TDOA. Sharing our experience with the readers, we are providing a summarized table which is comparing the existing methods.

The remainder of this paper is organized as follows. Section 2 explains the concept underlying operation of the system and its various parts, and outlines a relevant use case. Section 3 presents the mathematical derivation of the signal processing formulae. Section 4 outlines the simulations and experiments conducted and the results obtained. Section 5 discusses the sensitivity of the results to different system parameters. Section 6 presents concluding remarks.

\section{Background}

The proposed system is a passive RF sensing system that receives RF signals originating from an enclosed room. The source located in the room may be the network communication signal of a cellular phone or its Bluetooth or Wi-Fi signals. Metallic objects in the room reflect the source signal and their resulting echoes are externally sensed by an array of antennas that can detect the three-dimensional location of these metallic objects in the room.

To explain the operation of the system, we use the case of a Nokia 6210 cellphone operating at $900 \mathrm{MHz}$ Global System for Mobile Communication (GSM I), which is equivalent to an RF wavelength of $33 \mathrm{~cm}$ as the source. The cellular waveform and periodic time are illustrated in Figs. $1(\mathrm{a}-\mathrm{c})$. The detection range is approximately $10 \mathrm{~m}$ (determined experimentally). Once every few milliseconds, the cellphone located in the room, emits a string of RF signals (having an uplink with the master cell) that are described in the interactive protocols presented by Wu et al. (1997), Saunders \& Aragon-Zavala (2007) and Tse \& Viswanath (2005). The string duration is in the order of $1 \mathrm{~ms}$ and it contains multiple pulses having a pulse width of approximately $50 \mu \mathrm{s}$.

The original signal is detected with a delay of 10 to $30 \mathrm{~ns}$ (at the speed of light, it requires approximately 3 to $4 \mathrm{~ns}$ for it to travel one meter). The reflected signal travels an additional 2 to $8 \mathrm{~m}$ and is detected only 7 to 28 ns after the original signal based on its backreflection (echo) path. The time delay can indicate the depth of the reflecting object relative to the original source, as illustrated in Fig. 2.

For full coverage of a room with an area of $3 \times 6 \mathrm{~m}$, a matrix detection array comprising $15 \times 25$ elements is required (Chan \& Ho, 1994). With respect to the vertical

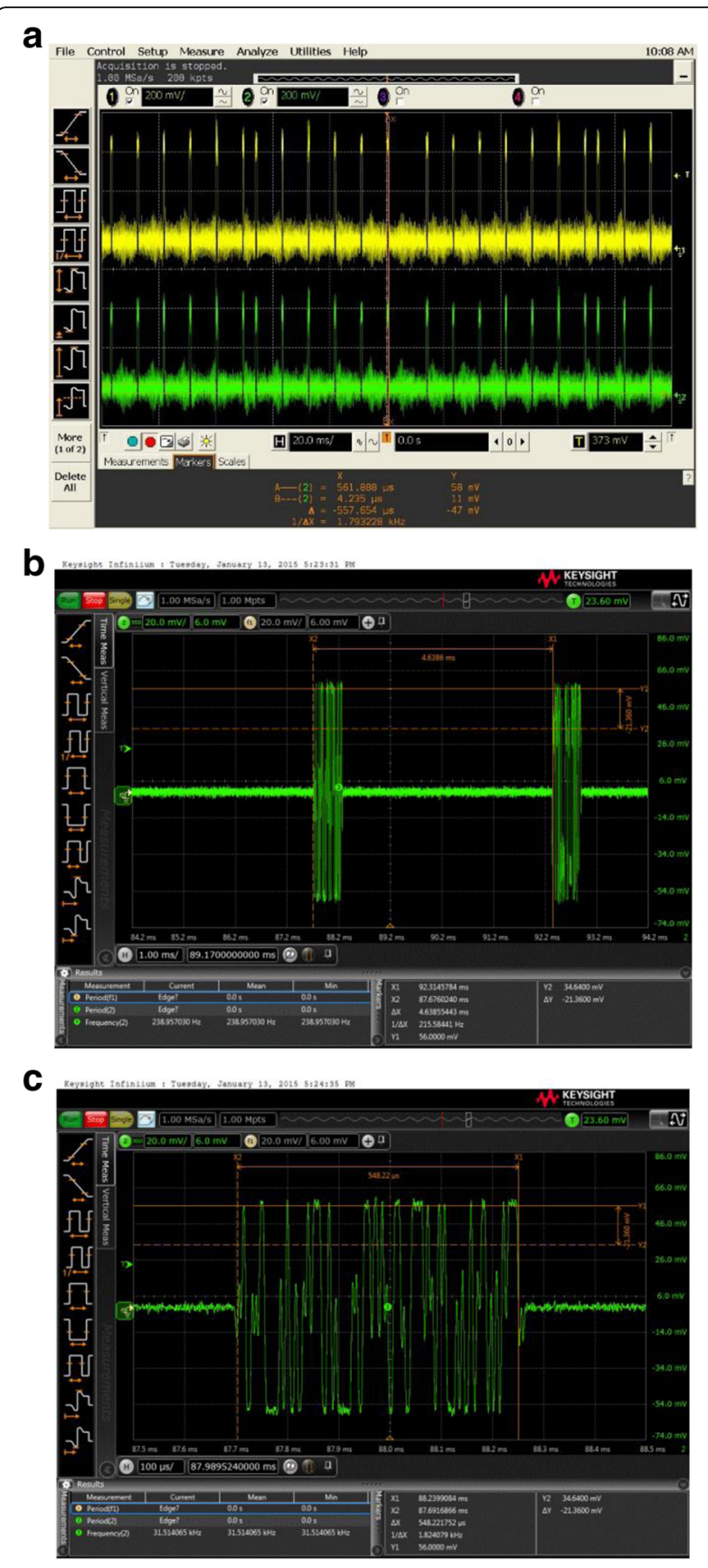

Fig. 1 a: Cellular protocol waveform; (b) two adjacent burst pulses; (c) intra-pulse modulation

axis, less than 15 elements can be used because the area of interest is covered by ten elements (Warren, 2008).

As the temporal length of the pulse is less than $1 \mathrm{~ms}$, it is assumed that a scanning system is not applicable unless each point is scanned within a different string. This scanning configuration requires several minutes for the complete the detection process. The accuracy of the time measurements must be better than $0.5 \mathrm{~ns}$ (0.1 ns recommended) to enable accurate mapping of the room 


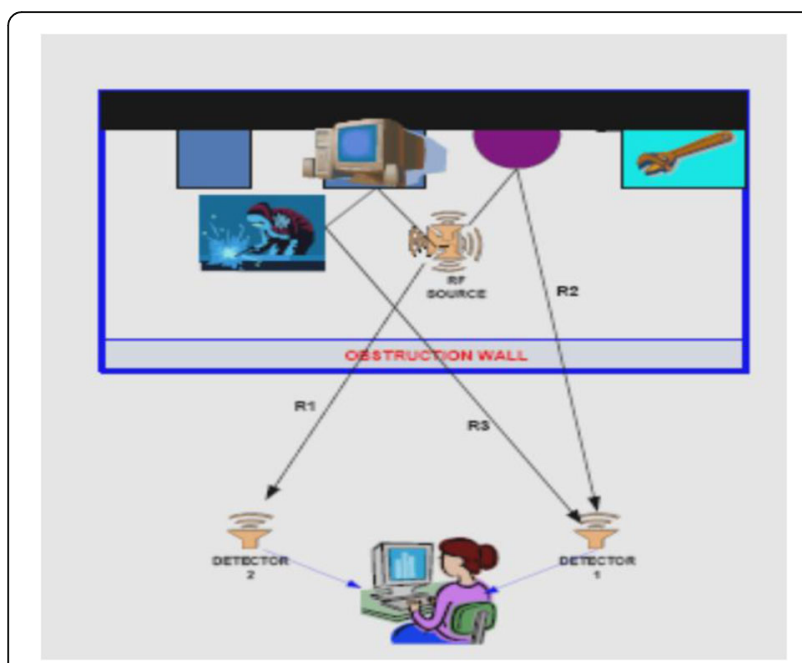

Fig. 2 Back scattering paths of electromagnetic waves to the detectors

layout when measuring the time delay between the reception of the original pulse and its back-reflected versions (Fang, 1990; Lee, 1989). The delay for a depth of $15 \mathrm{~cm}$ is less than $1 \mathrm{~ns}$ for complete two-way travel.

The proposed method employs the TDOA technique, a state of the art solution for this problem type. Signal processing is performed by a digital receiver (digitizer), which includes an input filter (pass-band $=1000 \pm$ $25 \mathrm{MHz}$ ), a logarithmic amplifier, and a digital processing block for the pulse width, amplitude, frequency, and time measurements. The graphical user interface of the digitizer is shown in Fig. 3, which shows a measured frequency of $1000 \mathrm{MHz}$. Most of the measured time delays were found to be approximately 1-4 ns. However, some were approximately $20 \mathrm{~ns}$, which indicates that multipathing occurred that is, part of the detection signals travels a longer path owing to the absence of a LOS.

\section{Method}

For the signal processing part of the experiment, the mathematical derivation comprises two aspects. (1) time-delay derivation between the arrival times at each receiver, which is derived by the cross correlation of the signals. (2) DDPhase derivation between any two receivers, which is carefully extracted using a phasemeasurement device, such as the procedure in a vector network analyzer (Table 1).

\section{Time-delay measurements}

Figure 4 depicts a rectangular coordinate system with a target located at $\left(x_{t}, y_{t}, z_{t}\right)$, the reference receiver at $(0,0,0)$, and $n$ receivers at $\left(x_{i}, y_{i}, z_{i}\right), i=1,2,3, \cdots, n$, where ${ }_{t} R$ is the distance between the reference receiver the target receiver, $R_{t}$ represents the distance between the reference receiver and receiver $i$ and $S_{i}$ denotes the distance between the target and receiver $i$.

Definition 1:

$$
\begin{aligned}
& R_{i}^{2}=x_{i}^{2}+y_{i}^{2}+z_{i}^{2}, i=1,2,3, \cdots, n \\
& R_{t}^{2}=x_{t}^{2}+y_{t}^{2}+z_{t}^{2} \\
& S_{i}^{2}=\left(x_{t}-x_{i}\right)^{2}+\left(y_{t}-y_{i}\right)^{2}+\left(z_{t}-z_{i}\right)^{2} \\
& \quad i=1,2,3, \cdots, n
\end{aligned}
$$

As $\Delta t_{i}=\left(R_{t}-S_{i}\right) / v$ for $i=1,2,3, \cdots, n, \Delta r_{i}=\left(R_{t}-S_{i}\right)$ where $v$ is the velocity of propagation. $\left(\Delta t_{i} s\right.$ are computed by cross correlation of the received signals at the receiver inputs).

The $\left(x_{t}, y_{t}, z_{t}\right)$ coordinates are computed from these $\Delta t_{i}^{\prime} s$ in (Eq. 1) as follows:

$$
R_{t}^{2}-S_{i}^{2}=x_{t}^{2}-\left(x_{t}-x_{i}\right)^{2}
$$

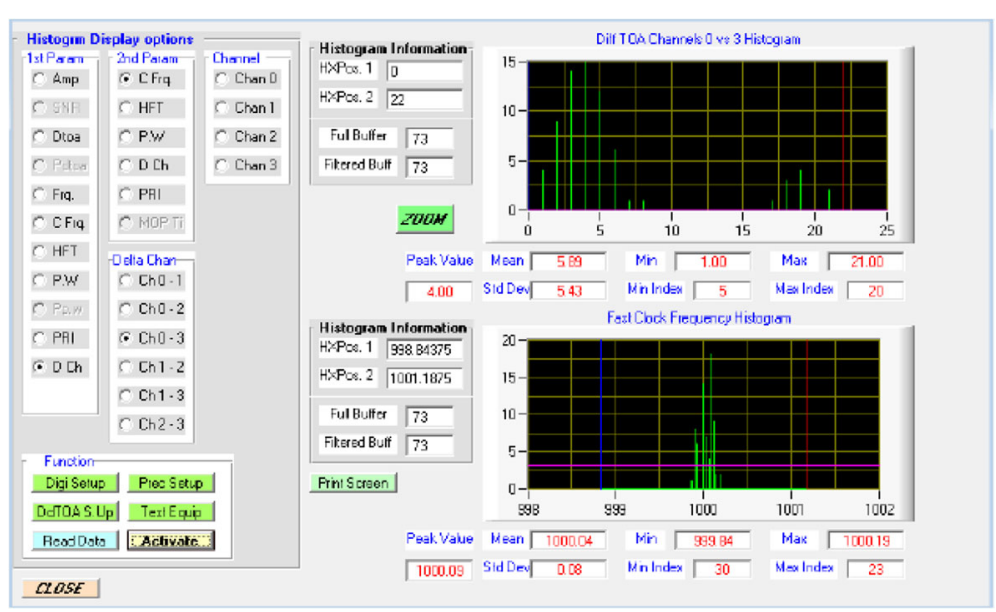

Fig. 3 Graphical user interface (frequency, pulse width (PW), pulse repetition interval (PRI), and TDOA) 
Table 1 Direction-finding measurement techniques comparison

\begin{tabular}{|c|c|c|c|c|c|}
\hline \multirow[b]{2}{*}{ Technique } & \multicolumn{5}{|l|}{ Parameter } \\
\hline & $\begin{array}{l}\text { Number of received } \\
\text { elements }\end{array}$ & $\begin{array}{l}\text { Distance between } \\
\text { elements }\end{array}$ & $\begin{array}{l}\text { Target relative } \\
\text { distance }\end{array}$ & $\begin{array}{l}\text { Azimuth and Elevation } \\
\text { Beam-width }\end{array}$ & Ambiguity \\
\hline Amplitude comparison & $2-3$ & Long & Medium-High & Narrow & High \\
\hline Interferometer & $4-5$ & Short & Medium & Narrow & Medium \\
\hline TOA & $3-4$ & Long & Medium-High & Wide & Medium \\
\hline TDOA & 4 & Short & Medium-High & Narrow & Medium \\
\hline DDPhase & 4 & Short-Long & Medium-High & Narrow & Low \\
\hline
\end{tabular}

$$
+y_{t}^{2}-\left(y_{t}-y_{i}\right)^{2}+z_{t}^{2}-\left(z_{t}-z_{i}\right)^{2}
$$

The left side of Eq. (2) becomes:

$$
\left(R_{t}+S_{i}\right)\left(R_{t}-S_{i}\right)=\left(2 R_{t}-\Delta r_{i}\right) \Delta r_{i},
$$

whereas the right side reduces to:

$$
2 x_{t} x_{i}+2 y_{t} y_{i}+2 z_{t} z_{i}-R_{i}^{2}
$$

Combining Eqs. (3) and (4) we get the following:

$$
x_{t} x_{i}+y_{t} y_{i}+z_{t} z_{i}=R_{t} \Delta r_{i}+\left(R_{i}^{2}-\Delta r_{i}^{2}\right) / 2
$$

Letting:

$$
\begin{aligned}
W_{i} & =\left(R_{i}^{2}-\Delta r_{i}^{2}\right) / 2, i=1,2,3, \cdots, n \\
W^{T} & =\left[W_{1}, W_{2}, \cdots, W_{n}\right] \\
D^{T} & =\left[\Delta r_{1}, \Delta r_{2}, \cdots, \Delta r_{n}\right] \\
V^{T} & =\left[x_{t}, y_{t}, z_{t}\right] \\
A & =\left[\begin{array}{ccc}
x_{1} & y_{1} & z_{1} \\
x_{2} & y_{2} & z_{2} \\
\cdot & \cdot & \cdot \\
x_{n} & y_{n} & z_{n}
\end{array}\right]
\end{aligned}
$$

Eq. (5) can thus be rewritten as follows:

$$
A V=R_{t} D+W
$$

Define, $B=\left(A^{T} A\right)^{-1} A^{T}$ (where $A$ should be of rank 3), $C=B D$ and $Y=B W$. Thus we have

$$
V=R_{t} C+Y
$$

Eq. (8) cannot be solved directly as $\left(x_{t}, y_{t}, z_{t}\right)$ occur in the right side of Eq. (2).

However,

$$
V^{T} V=R_{t}^{2} C^{T} C+R_{t}\left(C^{T} Y+Y^{T} C\right)+Y^{T} Y
$$

Thus, noting that $V^{T} V=R_{t}^{2}$,

$$
R_{t}^{2}\left(C^{T} C-1\right)+R_{t}\left(C^{T} Y+Y^{T} C\right)+Y^{T} Y=0
$$

The quadratic equation can thus be solved for $R_{t}$. Once $R_{t}$ is known, Eq. (8) can be solved $V^{T}=\left[x_{t}, y_{t}, z_{t}\right]$.

Thus, Eqs. (8) and (10) constitute an exact solution for the range computation problem.

\section{Delta-Delta phase}

Figure 5 shows an airborne Electronic Support Measures (ESM) system for direction finding with four receiving sensors and a target located at a spatial point $E(x, y, z)$. The system consists of four spiral antennas at the edge points of the platform that can receive

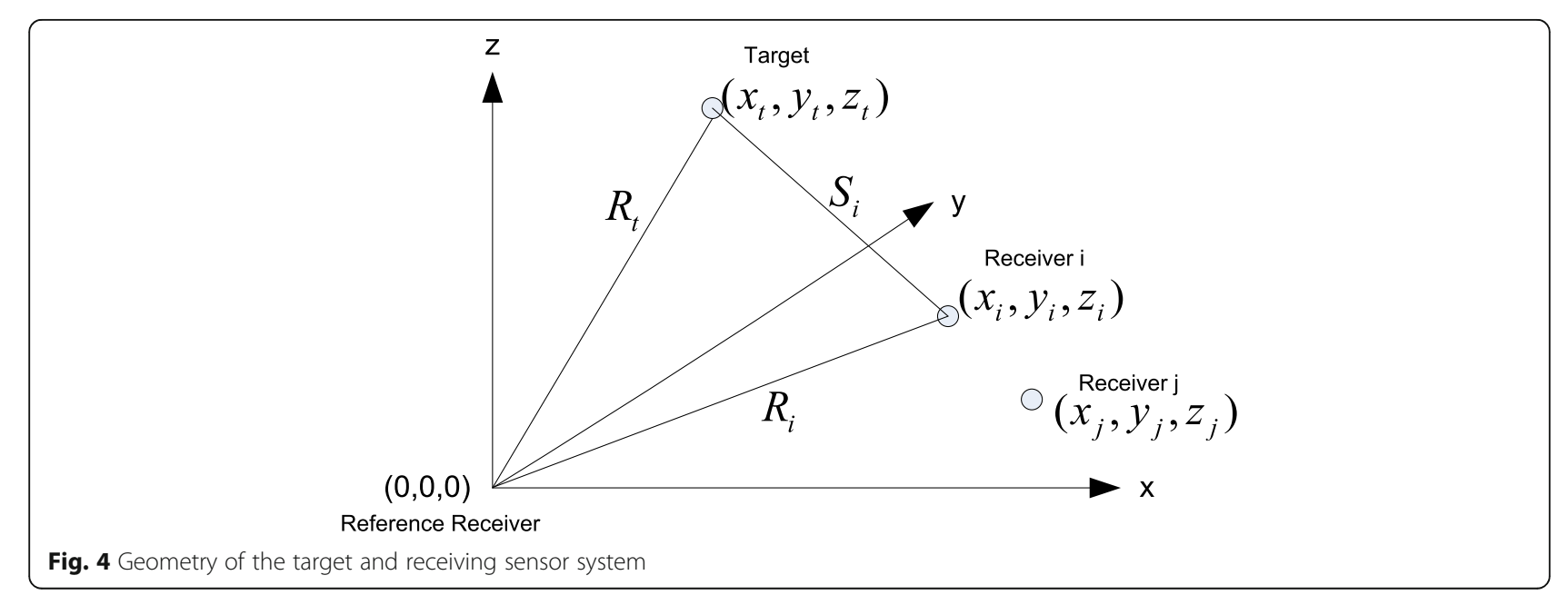




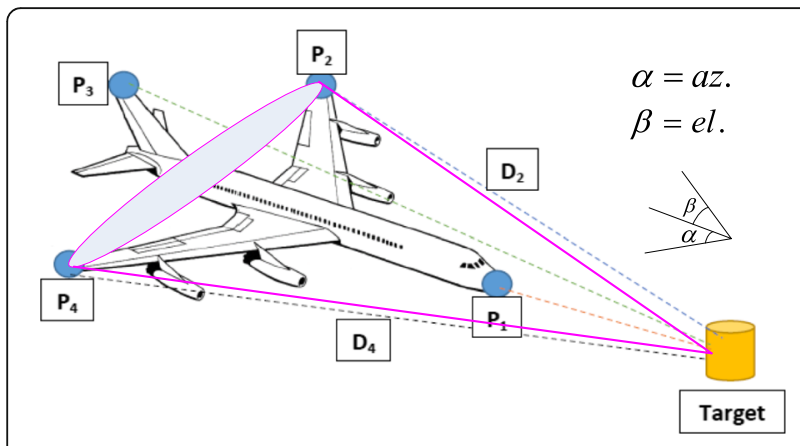

Fig. 5 Airborne ESM system for direction finding

signals from the air or ground to compute the angle of arrival.

The measured phase difference between any two sensors (i.e., $k$ and $j$ ) is as follows:

$$
\begin{aligned}
\Delta \phi_{j-k} & =\frac{2 \cdot \pi \cdot\left(\left\|P_{k}-E\right\| \cdot-\left\|P_{j}-E\right\|\right.}{\lambda} \\
& =\frac{2 \cdot \pi \cdot f}{c} \cdot\left(\left\|P_{k}-E\right\|-\left\|P_{j}-E\right\|\right),
\end{aligned}
$$

where.

$P_{j}, P_{k}$ are the respective locations of sensors $j$ and $k$.

The distances $D_{j}$ and $D_{k}$ between the target and any two sensors are respectively given by.

$$
D_{j}=\left\|P_{J}-E\right\| \text { and } D_{k}=\left\|P_{k}-E\right\|,
$$

where.
$E$ is the target location in Cartesian coordinates such that $E=\left(\begin{array}{lll}x_{E} & y_{E} & z_{E}\end{array}\right)^{T}$.

$f$ is the operating frequency $[H z]$.

$\lambda$ is the signal wavelength $[m]$.

$c$ is the speed of light $\left[3 \cdot 10^{8} \frac{\mathrm{m}}{\mathrm{sec}}\right]$.

The change in the phase difference between any two sensors $j$ and $k$ after a certain time is given by:

$$
\begin{gathered}
\Delta \Delta \phi_{j-k}=\frac{2 \cdot \pi \cdot f}{c}\left\{\left[\left(\left\|P_{k}(x+\Delta x)-E\right\|\right)-\left(\left\|P_{j}(x+\Delta x)-E\right\|\right)\right]\right. \\
\left.-\left[\left(\left\|P_{k}(x)-E\right\|\right)-\left(\left\|P_{j}(x)-E\right\|\right)\right]\right\}
\end{gathered}
$$

The same equations are written for each pair of antennas. The delta phase $(\Delta \phi)$ and the DDPhase between each pair of sensors are measured. The unit vector in the sensor direction with azimuth and elevation angles and the source is as follows:

$$
\frac{P-E_{j}}{\left\|P-E_{j}\right\|}=\left(\begin{array}{c}
\sin (\mathrm{az}) \cdot \cos (\mathrm{el}) \\
\cos (\mathrm{az}) \cdot \cos (\mathrm{el}) \\
\sin (\mathrm{el})
\end{array}\right)
$$

The geometry of all solutions of $E$ is a conical surface with an apex at point $S$ and a rotating axis that follows the length of cone $d$, where $d=\left\|P_{i}-P_{j}\right\|$, toward the base. The angle of tilt is $\alpha$, which approximately supports the equation $\Delta \phi_{i-j}=\frac{2 \pi \cdot f \cdot d}{c} \cos (\alpha)$. The final step is to use the principle of the maximum likelihood to estimate the target location, which is performed using MATLAB.

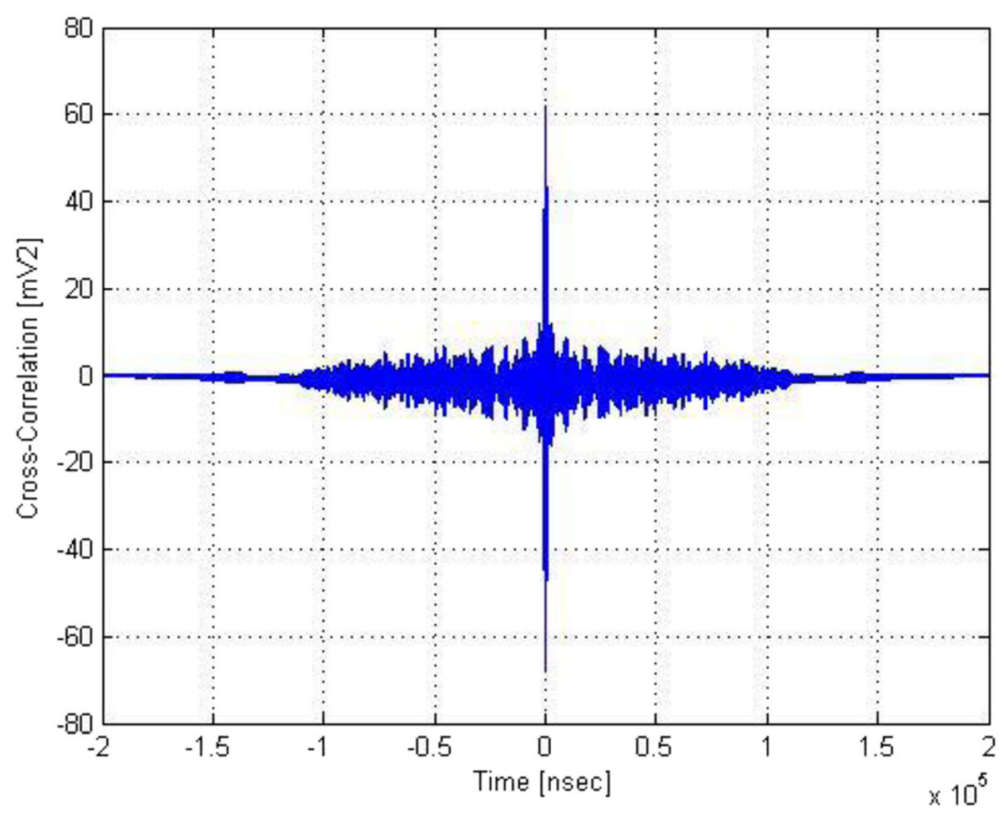

Fig. 6 Correlation pick as a result of convolution 


\section{Results and Discussion}

The experimental procedure was divided into three phases. In the first phase, a series of indoor measurements with controllable parameters was obtained. In the second phase, the main experimental phase, experiments were conducted outdoors in conditions very similar to those of the real operational scenario. In the third phase, targets were detected, including those behind concrete walls.

Indoor measurements in the laboratory (room dimensions $($ x w x h): $12 \times 5 \times 3 \mathrm{~m}$ )

A modulated signal generator was used as a source to create a pulsed waveform at $900 \mathrm{MHz}$ for reception by a four channel TDOA receiver. These measurements provided the true time delays between the channels. Further, the values obtained were ultimately used in the TDOA algorithm MATLAB code, which was based on the implementation of the typical cross-correlation technique shown in Fig. 6.

The following parameters were used:

- Frequency: $900 \mathrm{MHz}$

- Pulse width (PW):1 1 s

- Pulse repetition interval (PRI):10 $\mu \mathrm{s}$

- Cellphone model: Nokia 6210

- Four GSM Omni-antennas located at (x,y) coordinates $(1,2),(2,10.5),(4.5,8.5)$ and $(4.5,1.5)$ with

the transmitting source located at $(4,9)$.

With Ch\#1 normalized to $0 \mathrm{~ns}$, the values measured for the TDOA of the channels were as follows:

- $\mathrm{Ch} \# 2$ to $\mathrm{Ch} \# 1: 3.5 \mathrm{~ns}$

- Ch\#3 to Ch\#1: 15.5 ns

- Ch\#4 to Ch\#1: $32.4 \mathrm{~s}$

- Sampling scope with a rate of $10 \mathrm{Gbp} / \mathrm{s}$

- RF cables lengths: 2.2, 6.16, 17, and $17.74 \mathrm{~m}$

The measurement locations for the experimental setup are shown in Fig. 7(a). The results of the simulation code provide the exact location of the source, which is located at $(4,9)$, as shown in Fig. 8. (In the figure, some "detected points" exist owing to the leg of the metallic chair.)

\section{Outdoor measurements in the field (field dimensions:}

$50 \times 35 \mathrm{~m}$ )

A modulated signal generator source was set up using a signal generator and a wave modulation instrument to create a pulsed waveform at $900 \mathrm{MHz}$ for reception by the four channels of the TDOA receiver. These measurements provided the through-time delays between the channels that were subsequently used in the TDOA

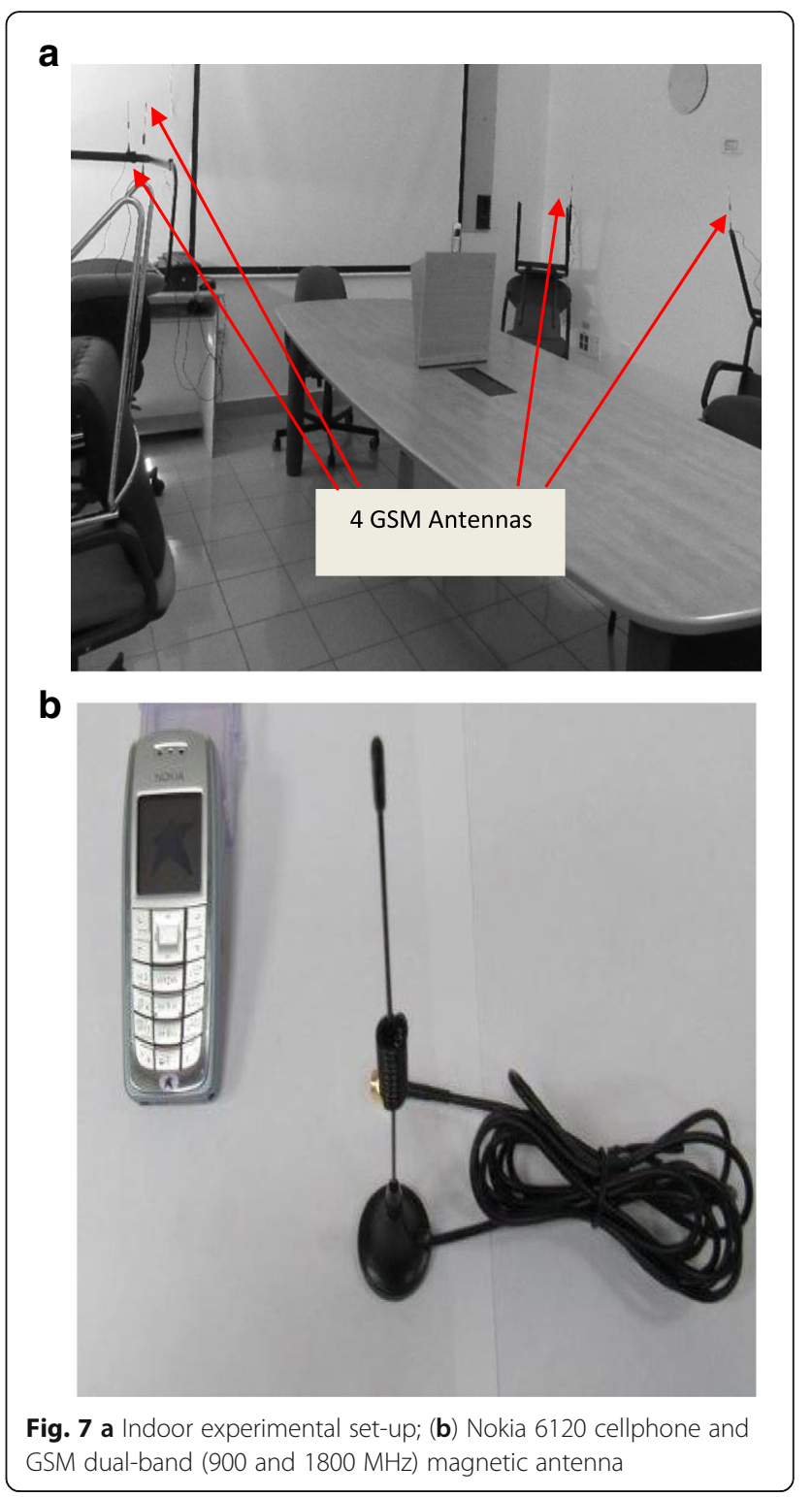

algorithm of the appropriate MATLAB code. The following parameters used were:

- Frequency: $900 \mathrm{MHz}$

- Pulse width (PW): $1 \mu \mathrm{s}$

- PRI: $10 \mu \mathrm{s}$

- Cellphone model: Nokia 6210

Four GSM Omni antennas located at the geographic (GEO) coordinates:

- E34.665626; N31.79323 (antenna reference)

- E34.665674; N31.79343

- E34.665692; N31.79318 


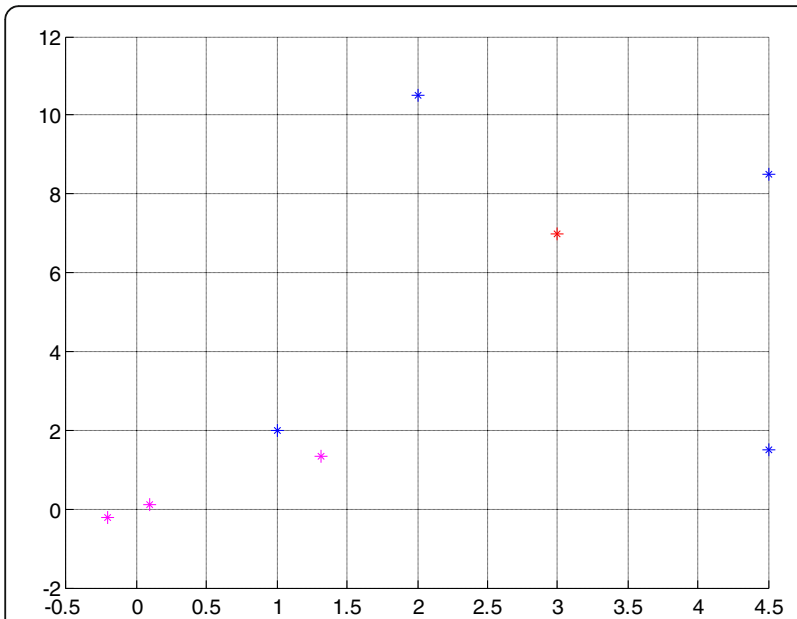

Fig. 8 Indoor measurement results (red star is the source location; pink stars are errors)

\section{- E34.665502; N31.79338}

The source was located at E34.665618; N31.79336.

With $\mathrm{Ch} \# 1$ normalized to $0 \mathrm{~ns}$, the values measured for the TDOA of the channels were as follows:

- $\mathrm{Ch} \# 2$ to $\mathrm{Ch} \# 1: 32.3$ ns

- $\mathrm{Ch} \# 3$ to Ch\#1: $56.4 \mathrm{~ns}$

- Ch\#4 to Ch\#1: 67.2 ns

- Modulated signal generator: Keysight E8663D

- Sampling scope with a rate of $10 \mathrm{Gbp} / \mathrm{s}$

- RF cables lengths: $6.2,23,38$ and $24 \mathrm{~m}$

The experimental setup is shown in Figs. 9(a-c). The results of the simulation code provided the exact location of the source, which was located at $(0,0)$, but as a normalized location, as shown in Fig. 10(a). In the subsequent experiment, two metallic plates were situated, as shown in Fig. 9(b). For this setup, the received signals were a mix of the signals received directly from the source and those reflected from the metallic plates. Using DDPhase processing, the time delays of the reflected signals were classified in order to compute the location of the sources. The results of the MATLAB code are shown in Fig. 10(b).

\section{Outdoor measurements with indoor RF sources and outdoor receiving antenna array}

The measurements were obtained for a reference localization of the sensors. The transmitting signals were received by the antennas and routed to the four inputs of the receiver. These measurements provided the phase value of each channel. The shelter had dimensions $8 \times$ $4 \times 2.5 \mathrm{~m}$ with a wall width of $20 \mathrm{~cm}$. The measurements were again obtained for another localization $(50 \mathrm{~cm}$
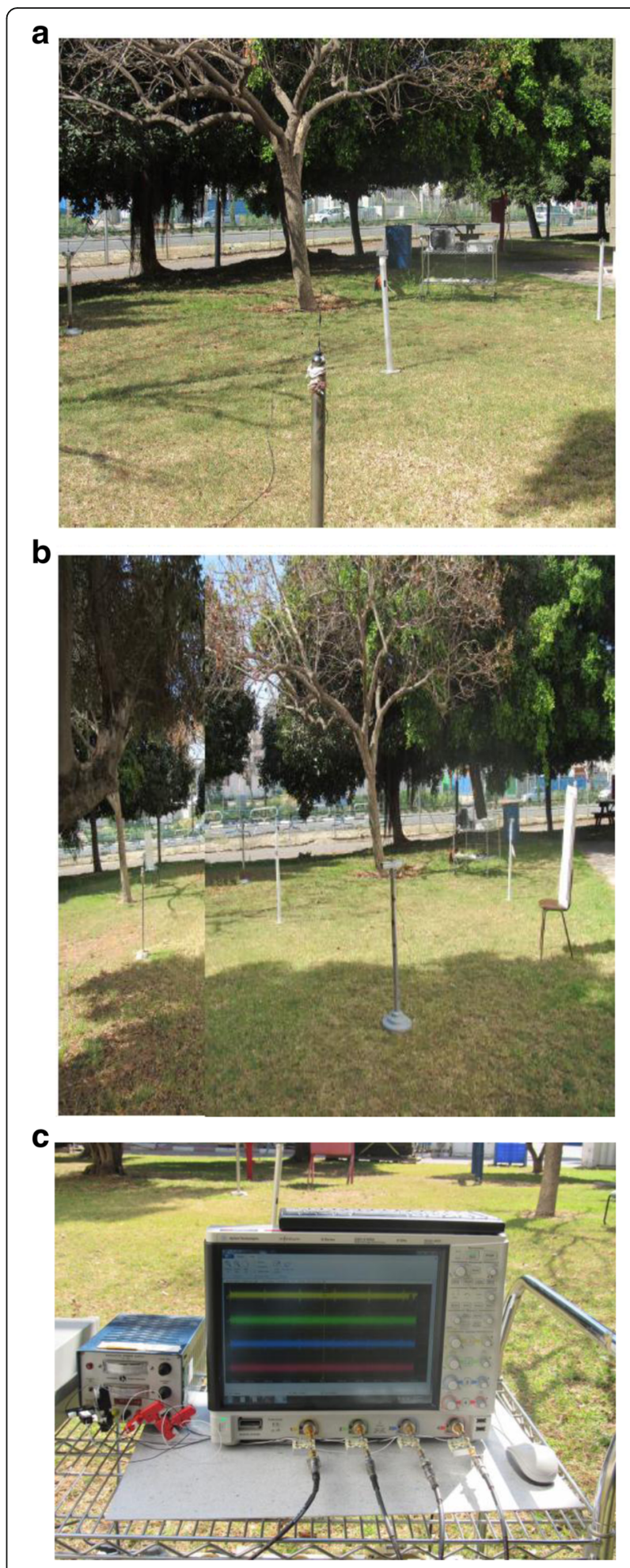

Fig. 9 Outdoor (a) setup without reflectors; (b) setup with reflectors; (c) measurements with the digitizer receiver

movement) of the sensors. The phase of each of the four channels was once again also measured. Using the DDPhase algorithm, the source location in the shelter 


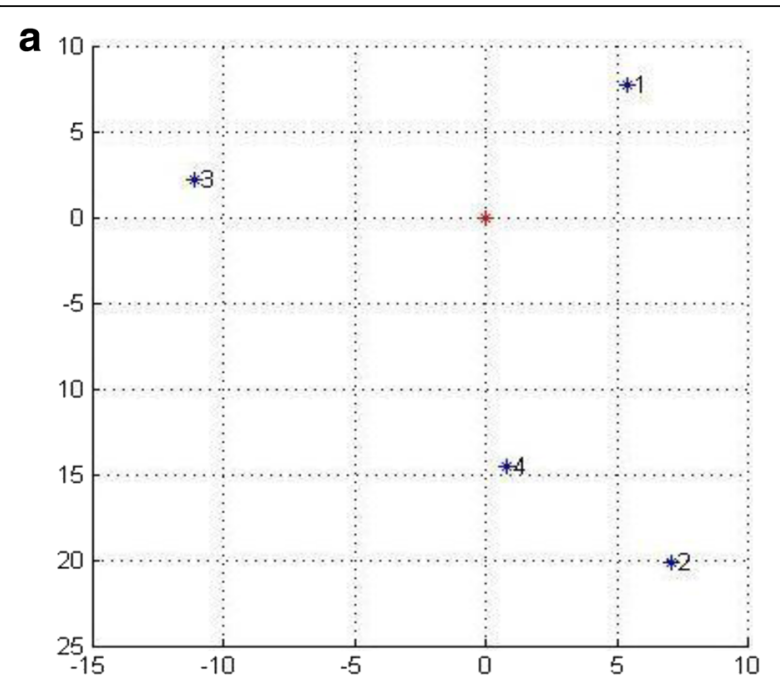

b

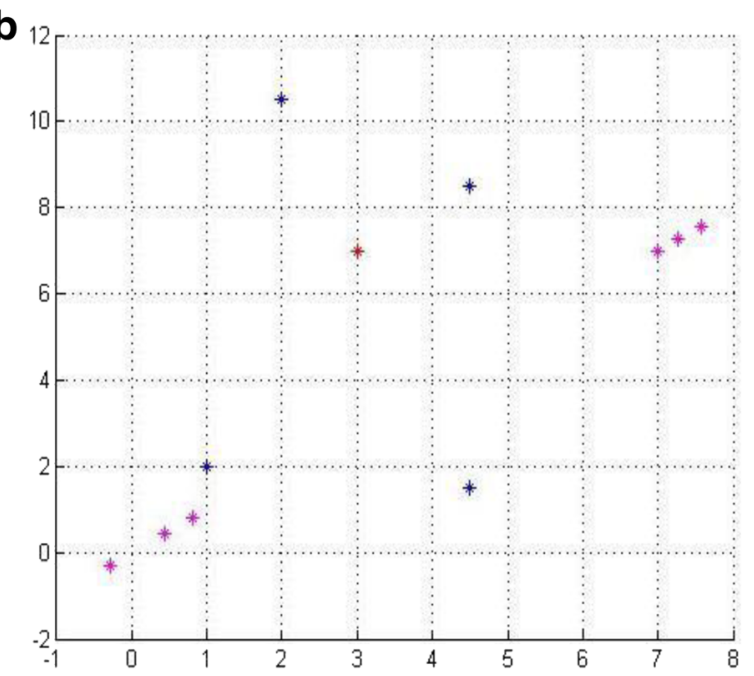

Fig. 10 Results: (a) without reflectors; (b) with reflectors (red star is the source location; pink stars are reflector locations)

was determined, as shown in Fig. 12. The following parameters were used:

- Cellphone model: Nokia 6210

- Four GSM Omni antennas located at (x, y) coordinates $(2,4),(4,5),(4,8)$ and $(4,10)$ respectively,

with the transmitting source located at $(4,9)$.

The measured values for the DDPhase of the channels were as follows:

- $\mathrm{Ch} \# 1$ at $\mathrm{t}=\mathrm{t} 1: 12^{\circ} \mathrm{Ch} \# 1$ at $\mathrm{t}=\mathrm{t} 2: 15^{\circ}$

- $\mathrm{Ch} \# 2$ at $\mathrm{t}=\mathrm{t} 1: 15^{\circ} \mathrm{Ch} \# 2$ at t $=\mathrm{t} 2: 20^{\circ}$

- $\mathrm{Ch} \# 3$ at $\mathrm{t}=\mathrm{t} 1: 15^{\circ} \mathrm{Ch} \# 3$ at $\mathrm{t}=\mathrm{t} 2: 20^{\circ}$

- $\mathrm{Ch} \# 4$ at $\mathrm{t}=\mathrm{t} 1: 12^{\circ} \mathrm{Ch} \# 4$ at $\mathrm{t}=\mathrm{t} 2: 15^{\circ}$

- Sampling scope with a rate of $10 \mathrm{Gbp} / \mathrm{s}$

- RF cables lengths: $3,4,4$ and $3 \mathrm{~m}$
The experimental setup is shown in Figs. 11(a, b). The RF source (cellphone) was indoors, as shown in Fig. 11(a), while the four receiving antennas were located outdoors in the same line as the shelter, as shown in Fig. 11(b). The results of the simulation code provided the exact location of the source, as shown in Fig. 12. Subsequent experiments were conducted under the same conditions as those mentioned above.

The data collected at the receivers were analyzed to determine the target locations. The apparatus used had a sampling rate of $10 \mathrm{GHz}$, which we deemed sufficient for this study. However, a higher sampling rate may give improved results, because as high sampling rate as high resolution, means more sampled points in a certain period. The analysis was initiated using typical localization algorithms, however, these were

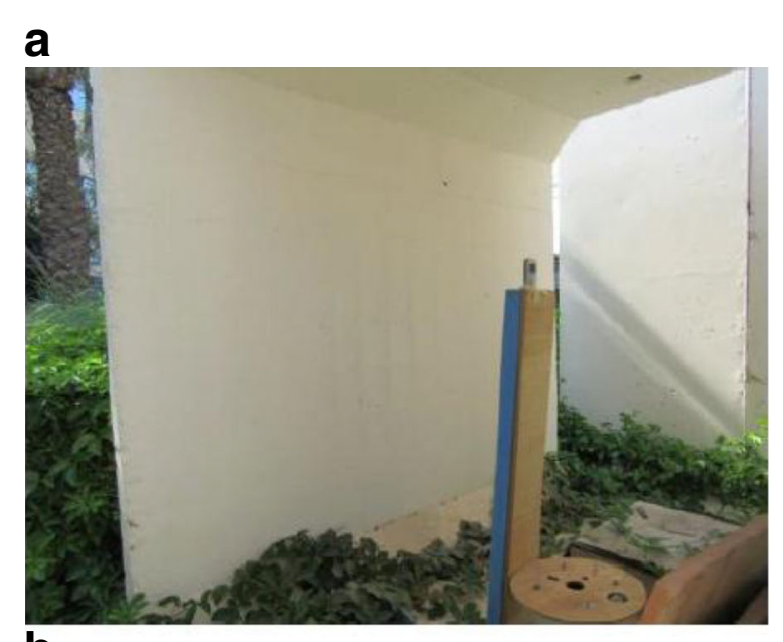

b

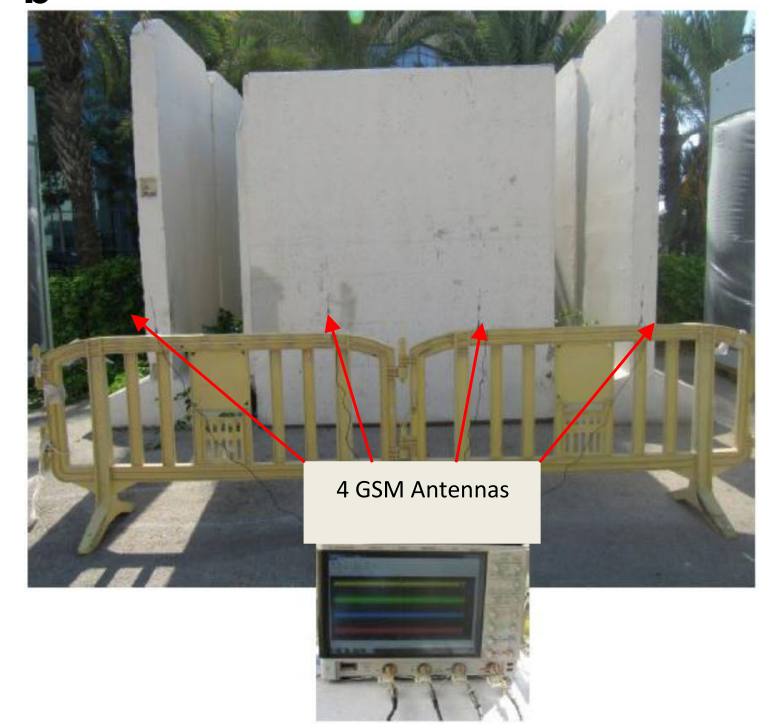

Fig. 11 Outdoor experimental setup: (a) indoor GSM cellphone; (b) outdoor antenna array 


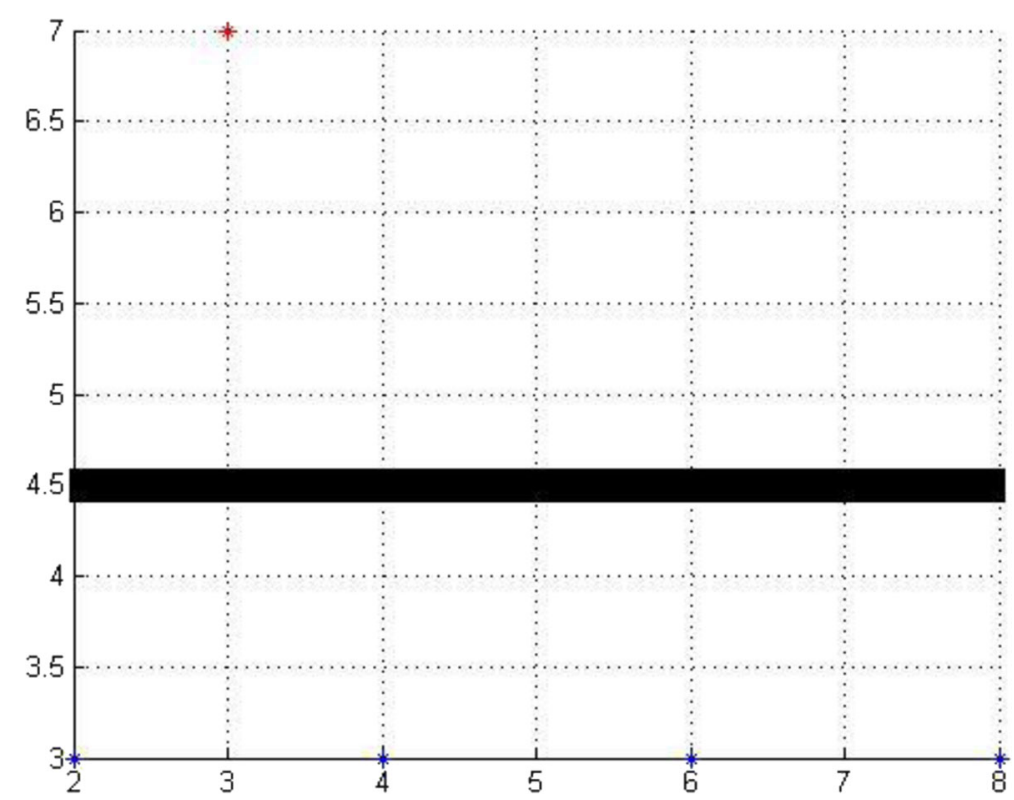

Fig. 12 Measurement results of the $20 \mathrm{~cm}$ thick concrete wall (red star is the source location; blue stars are the four receiving antennas; wide black line is the shelter wall)

found to be unsuitable because they not provide a reasonable accuracy less than $0.5 \mathrm{~m}$. By testing three different setups of the source location and sensor locations, the optimal technique for improving the measurement accuracy of the target localization was determined. By using an optimal MATLAB code which based on geometrical calculation to preserve the important parameters for determining the target location to find the effects of the spectrum background noise on measurements. The optimal technique for improving the measurement accuracy of the target localization was determined. A general comparison of measurement techniques and locations is shown in Table 2 below.

Several advanced algorithms based on phase measurement techniques and changes in the phase differences were employed to extract the essential parameters. The measured phase at time $t=t_{i}$ from any route $R_{j}$ is $P h_{j}\left(t_{i}\right)$, where $i$ is a synchronized time base and $j$ and $k$ are any two of the four receiving elements.

The differential phases at time $t=t_{i}$ between routes $R_{j}$ and $R_{k}$ is $\Delta P h_{j-k}=P h_{k}-P h_{j}$. The value of the DDPhase between

Table 2 Direction-finding measurement techniques and location comparison

\begin{tabular}{llll}
\hline $\begin{array}{l}\text { Case } \\
\text { [Section 4] }\end{array}$ & $\begin{array}{l}\text { Technique } \\
\text { [Processing] }\end{array}$ & $\begin{array}{l}\text { Source / Sensor } \\
\text { Locations }\end{array}$ & $\begin{array}{l}\text { Accuracy } \\
{[\mathrm{m}]}\end{array}$ \\
\hline Part A & TDOA & In / In & 0.5 \\
Part B & TDOA & Out / Out & 1 \\
Part C & DDPhase & In /Out & 0.3 \\
\hline
\end{tabular}

$t=t_{1}$ and $t=t_{2}$ is related to the routes traveled between $R_{j}$ and $R_{k}$ and is given by: $\Delta P h_{j-k}\left(t_{2}\right)-\Delta P h_{j-k}\left(t_{1}\right)$.

For stationary targets, or when the velocity of the target is low, this algorithm provides the location with accuracy in the order of 10 to $20 \mathrm{~cm}$. The accuracy deteriorates if the velocity becomes higher and/or if the line of sight to the target significantly changes. Another parameter that affects the accuracy is the relative distance between the different receivers (the base line) relative to the target. This accuracy is achieved for relative distances that create an angular line-of-sight separation in the order of several tens of degrees. As the phase change is significant, which is the case for moving target, we get better accuracy.

For the present analysis, it was assumed that the walls covering the target area neither significantly deflected the radiation nor attenuated the signals. This assumption is applicable for many constructions; however, it is not suitable for metallic or reinforced concrete walls. To further improve the measurement accuracy, directional antennas, which enable better target resolution, can be used. The narrow beam width antennas enable separation of the signals that improve the measurement accuracy and resolve possible ambiguity.

\section{Conclusions}

In this paper, an RF echo mapping technique based on a direction-finding model was proposed. The method employs the concept of four cellular GSM Omni-antennas. This system was shown to be feasible and provided 
accurate results. The basic solution of the TOA computation was found to be satisfactory for indoor and static targets when the source was a stationary signal and unmodulated. However, in the case of outdoor and modulated signals, it was found to be very limited, in fact for moving targets, because of multi-pathing and the Doppler effect. The DDPhase technique proved useful for detecting targets behind walls. Ambiguity caused by multi-pathing was resolved by classifying the received signals and calculating their time delays using a digitizer that can measure fast and very narrow pulses in order to provide time delays with high accuracy (for example, $1 n s$ ). Conventionally, when the target is very small, the baseline between the received antennas is very short and measurement accuracy typically decreases. The proposed DDPhase method overcomes this problem for small targets and high speed targets with results that are accurate to within $0.3 \mathrm{~m}$ due to the high resolution of the phase measurements.

The experimental results reported in Section 4 are based on a transmitting pulsed signal of $900 \mathrm{MHz}$ because of the cellphone and antenna operating range. The same process can be used for lower or higher frequency ranges; however, an appropriate source and antenna are required. As the RF signals used in this study were cellular signals, Wi-Fi, Bluetooth, LTE and any modern cellular signals can be covered. Factors such as the signal-sampling rate, distances between the antennas relative to the distance to the source, and the measurement accuracy resulting from the conversion from frequency to wavelength, will affect the results computed.

\begin{abstract}
Abbreviations
AOA: Angle of Arrival; DDPhase: Delta Delta Phase; DOA: Direction of Arrival; ESM: Electronic Support Measures; Gbp/s: Giga bit per second; GHz: Giga Hertz; GSM: Global System for Mobile communication (Group Special Mobile); LOS: Line of Sight; MATLAB: Matrix Laboratory (Software); MHz: Mega Hertz; PRI: Pulse Repetition Interval; PW: Pulse Width; RF: Radio Frequency; TDOA: Time Difference of Arrival; TOA: Time of Arrival
\end{abstract}

\section{Acknowledgements}

Isahar Gabay is thankful to Prof. Zalevsky and Prof. Danino for their help and support during the writing of the manuscript. Also a great thanks to Editage (www.editage.com) for English language editing.

\section{Funding}

The authors declare no specific funding provided

\section{Availability of data and materials}

Non-social (i.e. locations and pictures) can be obtained by emailing the corresponding author at: gabayi@elta.co.il.

\section{Authors' contributions}

ZZ had the original idea for the application and partially laid out the original design. MD laid out the original design and the experiments set-up and results. IG had the implementation of the theory, wrote the MATLAB codes, made the experiments and organized the database, wrote the main text and created the figures. All authors contributed to the drafts and gave final approval for publication.

\section{Competing interests}

The authors declare no competing interests

\section{Publisher's Note}

Springer Nature remains neutral with regard to jurisdictional claims in published maps and institutional affiliations.

Received: 17 October 2017 Accepted: 17 January 2018

Published online: 13 February 2018

\section{References}

Brookner E. Practical phased array antenna systems. Artech house. 1991:85-102.

Chan YT, Ho KC. A simple and efficient estimator for hyperbolic location. IEEE Transactions on Signal Processing. 1994;42(8):1905-15.

Demmel F. Practical aspects of design and application of direction finding systems: Academic Press; 2009.

Fang BT. Simple solutions for hyperbolic and related position fixes. IEEE Transactions on Aerospace and Electronic Systems. 1990;26(5):748-53.

Gething PJD. Radio direction finding and super resolution. London: Peter Peregrinus, Ltd.; 1990.

Grosicki, E., \& Abed-Meraim, K. (2005). A new trilateration method to mitigate the impact of some non-line-of-sight errors in TOA measurements for mobile localization. In Acoustics, Speech, and Signal Processing, 2005. Proceedings. (ICASSP'05) (Vol. 4, pp. iv-1045).

Hansen RC. Phased array antennas. 2nd ed: Wiley; 2010. p. 186-232.

Lee WC. Spectrum efficiency in cellular (radio). IEEE Trans Veh Technol. 1989; 38(2):69-75.

Lim, J. S., Chae, G. S., \& Park, Y. C. (2005). A novel technology of microwave direction finding with a combination of amplitude measurement and phase measurement. Proceedings of ISAP.

Mailloux, R. J. (2005). Phased array antennas handbook. Artech house, 2nd Ed. , pp. 1-43.

Patwari N, Hero AO, Perkins M, Correal NS, O'dea RJ. Relative location estimation in wireless sensor networks. IEEE Trans Signal Process. 2003;51 (8):2137-48.

Rocca P, Morabito AF. Optimal synthesis of reconfigurable planar arrays with simplified architectures for monopulse radar applications. IEEE Trans Antennas Propag. 2015;63(3):1048-58.

Saunders RS, Aragon-Zavala A. Antenna and propagation for wireless communication systems: John Wiley \& Sons; 2007.

Tse D, Viswanath P. Fundamentals of wireless communication: Cambridge University Press; 2005

Tuncer TE, Friedlander B. (Eds.). Classical and modern direction-of-arrival estimation: Academic Press; 2009.

Wu JS, Chung JK, Sze MT. Analysis of uplink and downlink capacities for two-tier cellular system. IEEE Proceedings-Communications. 1997, December;144(6): 405-11.

\section{Submit your manuscript to a SpringerOpen ${ }^{\mathcal{O}}$ journal and benefit from:}

- Convenient online submission

Rigorous peer review

- Open access: articles freely available online

- High visibility within the field

- Retaining the copyright to your article

Submit your next manuscript at $>$ springeropen.com 